Research Paper

\title{
Smoking Induced Extracellular Vesicles Release and Their Distinct Properties in Non-Small Cell Lung Cancer
}

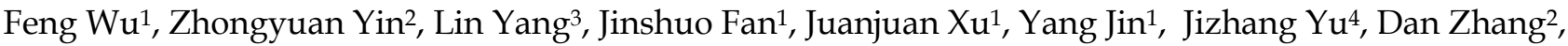 \\ Guanghai Yang ${ }^{\square}$ \\ 1. Department of Respiratory Medicine, Union Hospital, Tongji Medical College, Huazhong University of Science and Technology, Wuhan 430022, China \\ 2. Cancer Center, Union Hospital, Tongii Medical College, Huazhong University of Science and Technology, Wuhan 430022, China \\ 3. Oncology Department, Tongji Hospital, Tongji Medical College, Huazhong University of Science and Technology, Wuhan, 430030, China \\ 4. Department of Thoracic Surgery, Union Hospital, Tongji Medical College, Huazhong University of Science and Technology, Wuhan 430022, China
}

$\triangle$ Corresponding author: Guanghai Yang, Department of Thoracic Surgery, Union Hospital, Tongji Medical College, Huazhong University of Science and Technology, Wuhan 430022, China. Tel.: + 008627 85351615. Email: 2004xh0838@hust.edu.cn

(C) Ivyspring International Publisher. This is an open access article distributed under the terms of the Creative Commons Attribution (CC BY-NC) license (https://creativecommons.org/licenses/by-nc/4.0/). See http://ivyspring.com/terms for full terms and conditions.

Received: 2018.10.04; Accepted: 2019.04.23; Published: 2019.06.09

\begin{abstract}
Purpose: Smoking is a strong relative risk factor for lung cancer. Extracellular vesicles (EVs), particularly exosomes, have been implicated in cancers. In this study, we characterized smoking induced extracellular vesicles in smokers with non-small cell lung cancer (NSCLC).

Methods: EVs were isolated from bronchoalveolar lavage (BAL) from smokers and NSCLC patients. EV microRNAs (miRNAs) were analyzed by using a TaqMan microRNA assays. Vesicle mRNAs and long non-coding RNAs (IncRNAs) were measured with quantitative RT-PCR. Tumor associated antigens were examined by Western Blot.

Results: Higher levels of local site EVs are found in the lung of smokers and NSCLC patients. Further, over $90 \%$ of lung EVs are round vesicles of approximately 50-200 nm, ie., exosomes. There are $21 \mathrm{EV}$ miRNAs up regulated, while 10 miRNAs under regulated, in smokers when compared to controls (relative fold $>2, p<$ 0.05). These miRNAs were further observed to be dysregulated in NSCLC patients when compared to smokers. Bioinformatic analysis demonstrated that Proteoglycans, Fatty acid biosynthesis, ErbB, Hippo, TGF-beta, Wnt, Rapl, AMPK and Ras pathways were the most prominent pathways enriched in NSCLC EV miRNA signatures. In addition, messenger RNA transcripts including EGFR, KRAS, ALK, MET, LKBI, BRAF, PIK3CA, RET, and ROS1 were significantly higher expressed in lung EVs in smokers and NSCLC patients compared to controls. Long non-coding RNAs, including MALAT1, HOTAIR, HOTTIP, AGAP2-AS1, ATB, TCF7, FOXD2-AS1, HOXA11-AS, PCAF1, and BCAR4, were over expressed in EVs from smokers and NSCLC patients. Furthermore, protein levels of tumor associated antigens including BAGE, PD-L1, MAGE-3, and AKAP4 were significantly dysregulated in EVs of smokers and NSCLC patients compared to healthy controls.

Conclusions: In conclusion, these data demonstrated an intrinsic relationship of smoking dysregulated EVs and EVs contained RNA, proteins which may involve in the development of NSCLC.
\end{abstract}

Key words: extracellular vesicles, smoker, NSCLC, tumor associated antigens

\section{Introduction}

Smoking is by far the leading risk factor for lung cancer [1-4]. In the United States, cigarette smoking is linked to about $80 \%$ to $90 \%$ of lung cancers. Even people don't smoke; breathing in the smoke of others (called secondhand smoke or environmental tobacco smoke) can increase the risk of developing lung cancer. Secondhand smoke is further thought to cause more than 7,000 deaths from lung cancer each year [5, 6]. Non-small-cell lung carcinoma (NSCLC) accounts for about $85 \%$ of all lung cancers, which are relatively insensitive to chemotherapy compared to small cell carcinoma [1-4]. 
Recent studies indicated that membrane-bound extracellular vesicles (EVs; 30-400nm) are important mediators of intercellular communication among different tissues and organs [7]. EVs, found in most bodily fluids, contain proteins, RNA and lipids [7]. Exosomes $(\sim 50-200 \mathrm{~nm})$ are released through the fusion of multivesicular bodies with the plasma membrane [8]. Exosomes have a role in physiological and pathological intercellular signaling and in a wide spectrum of biological functions [8]. It is currently unknown how smoking affects lung EVs pattern and their microRNA (miRNA) expression. In vitro studies suggest that tobacco smoke increased the release of pro-coagulant tissue factor positive extracellular vesicles by monocytes and macrophages [9], while studies on human subjects rather indicated a decrease in plasma EVs [10, 11]. However, EVs are derived from many different cell types and vary extensively in size. Specifically, EVs encompassing the size range of exosomes, microvesicles and apoptotic bodies have not been investigated extensively in the context of smoking populations. Therefore, we determined the effects of habitual smoking on EVs profile in bronchoalveolar lavage (BAL) fluid and alterations in the NSCLC-related miRNAs, mRNAs, long non- coding RNAs (lncRNAs). We further examined differential expression of tumor associated antigens in EVs from smokers and NSCLC patients.

\section{Materials and Methods}

\section{Ethics statement}

This work received approval from the institution ethics committee of Tongji Medical College, Huazhong University of Science and Technology.

\section{Patients}

Clinicopathologic characteristics of smokers and lung cancer patients are summarized in Supplementary materials. Healthy subjects were patients undergoing endoscopy for unexplained upper abdominal complaints, had no reflux symptoms or endoscopic abnormalities, and did not use PPIs. Inclusion criteria for smoker patients required at least a 10 pack-year history of smoking, while the nonsmoker control subjects were self-reported never smokers. Smoking subjects were excluded if they had any significant comorbid conditions such as pregnancy or other acute or chronic disease such as pre-existing asthma, interstitial lung diseases or cardiovascular diseases. NSCLC patients have histologically or cytologically confirmed non-small cell lung cancer not amenable to curative intent therapy or stage IV NSCLC. Exclusion criteria includes patients who are concurrently receiving chemotherapy, biologics, immunotherapy for cancer treatment; as well as systemic anti-cancer treatment or investigational treatment. NSCLC subjects were also excluded if patients have a history of other malignancies within the last 5 years, except for non-melanoma skin cancer or carcinoma in situ of the cervix.

\section{Bronchoalveolar lavage}

BAL was performed by using standard protocol. The BAL consisted of 2 aliquots of $50 \mathrm{~mL}$ of normal saline instilled and aspirated manually using a $60 \mathrm{~mL}$ syringe. All subjects underwent conventional bronchoscopy. The BAL fluid was centrifuged at $1500 \mathrm{rpm}$ for 10 minutes at $4^{\circ} \mathrm{C}$. The supernatant was aliquoted was stored at $-80^{\circ} \mathrm{C}$ for further EVs analysis.

\section{Isolation of extracellular vesicles}

EVs were isolated as described before [12]. In brief, BAL fluid was subjected to successive centrifugations. EV pellets were resuspended in $0.32 \mathrm{M}$ sucrose and centrifuged at $100,000 \times \mathrm{g}$ for 1 hour.

\section{Extracellular vesicles characterization by FACS analysis}

BAL fluid was added directly to Dynabeads (2 $\mathrm{mL} / \mathrm{mL}$ beads) coated with anti-MHC class II (MHCII) antibodies (clone HKB1; Invitrogen/Dynal).

\section{RNA processing and MicroRNA expression profiling}

Total RNA was extracted by using a mirVana miRNA isolation kit (Applied Biosystems, Foster City, CA). TaqMan microRNA assays (Applied Biosystems) was utilized for vesicle miRNA profiling. DIANAmirPath [13] was introduced in biological pathways.

\section{RT-qPCR}

Total RNA quantity and quality was tested using the Agilent Bioanalyzer RNA 6000 Chip (Agilent, Santa Clara, CA). Reverse transcribed using random hexamers and reverse transcriptase and RT-qPCR was performed using gene-specific primers and SYBR green master mix on a 7900HT Fast Real-Time PCR System (Applied Biosystems). Gene expression was normalized to the average of HPRT.

\section{Immunoblotting}

EVs $(10 \mu \mathrm{g})$ samples were lysed and subjected to SDS-PAGE and immunoblotted with antibodies against HLA-class I, AKAP4, BAGE, PD-L1, MAGE-3, Annexin-A2, and Annexin V (Cell Signaling Technology).

\section{Statistical analysis}

Mann-Whitney test was used to compare gene expression levels. Data were shown as mean \pm SE. P < 0.05 were recognized statistically significant. 


\section{Results}

\section{Higher EV concentration with smoking}

To assess differences in lung EVs with smoking, we designed cross sectional cohorts including smokers and NSCLC patients. Isolated BAL EVs were validated according to the International Society of Extracellular Vesicles guidelines [14]. Known EV markers were present in each of the EV samples. The electron microscopy image shows intact, round vesicles of approximately 50-200 nm. A similar size distribution was also confirmed by nanoparticle tracking analysis (NTA) with a peak around $80 \mathrm{~nm}$ (Figure 1A). No significant differences were seen between the groups. These data confirm the size, morphology and protein markers that are characteristic of EVs.

We sought to determine whether there were differences in lung EV concentration between smokers and NSCLC patients. An analysis of a crosssectional cohort consisting of both groups revealed a significantly higher EV concentration in 14 smokers and 17 NSCLC patients compared to 14 healthy subjects (Smokers vs. Controls, $\mathrm{p}=0.022$; NSCLC vs. Controls, $\mathrm{p}=0.007$; Figure 1C). Clinicopathologic characteristics of smokers and NSCLC patients are summarized in Supplementary Table 1.

To further confirm our results obtained using precipitation isolation methods, we used a protocol recently reported by Kowal et al. to compare EVs recovered from different steps of the differential ultracentrifugation protocols [15]. Smokers and NSCLC patients had higher concentration of EVs isolated at both medium $(10,000 \times \mathrm{x})$ and ultracentrifugation $(120,000 \times \mathrm{g})$ speeds (Smokers vs. Controls, $p=0.015$; NSCLC vs. Controls, $p=0.001$; Figure 1D). Thus, smoking is associated with higher levels of local site EVs in the lung, which further indicated higher EV population, may play an important role in NSCLC development.

\section{Dysregulated microRNAs expression in lung EVs from smokers and NSCLC patients}

We profiled the expression of 366 miRNAs by using TaqMan miRNA arrays in an independent cohort of smokers and NSCLC patients (Supplementary Table 2). Vesicle miRNAs were normalized to the BAL volume. The results shown that 102 miRNAs (27.8\%) could be detected. Further, 31 vesicle miRNAs were differentially expressed in lung EVs from smokers compared to healthy subjects, including 21 up-regulated vesicle miRNAs and 10 under-regulated miRNAs $(p<0.05)$ (Table 1). Further, these 31 vesicle miRNAs shown similar expression change patterns in NSCLC exosomes when compared to those from healthy subjects (Table 1 and Figure 2). Further bioinformatics study demonstrated that 49 Kyoto Encyclopedia of Genes and Genomes (KEGG) path-
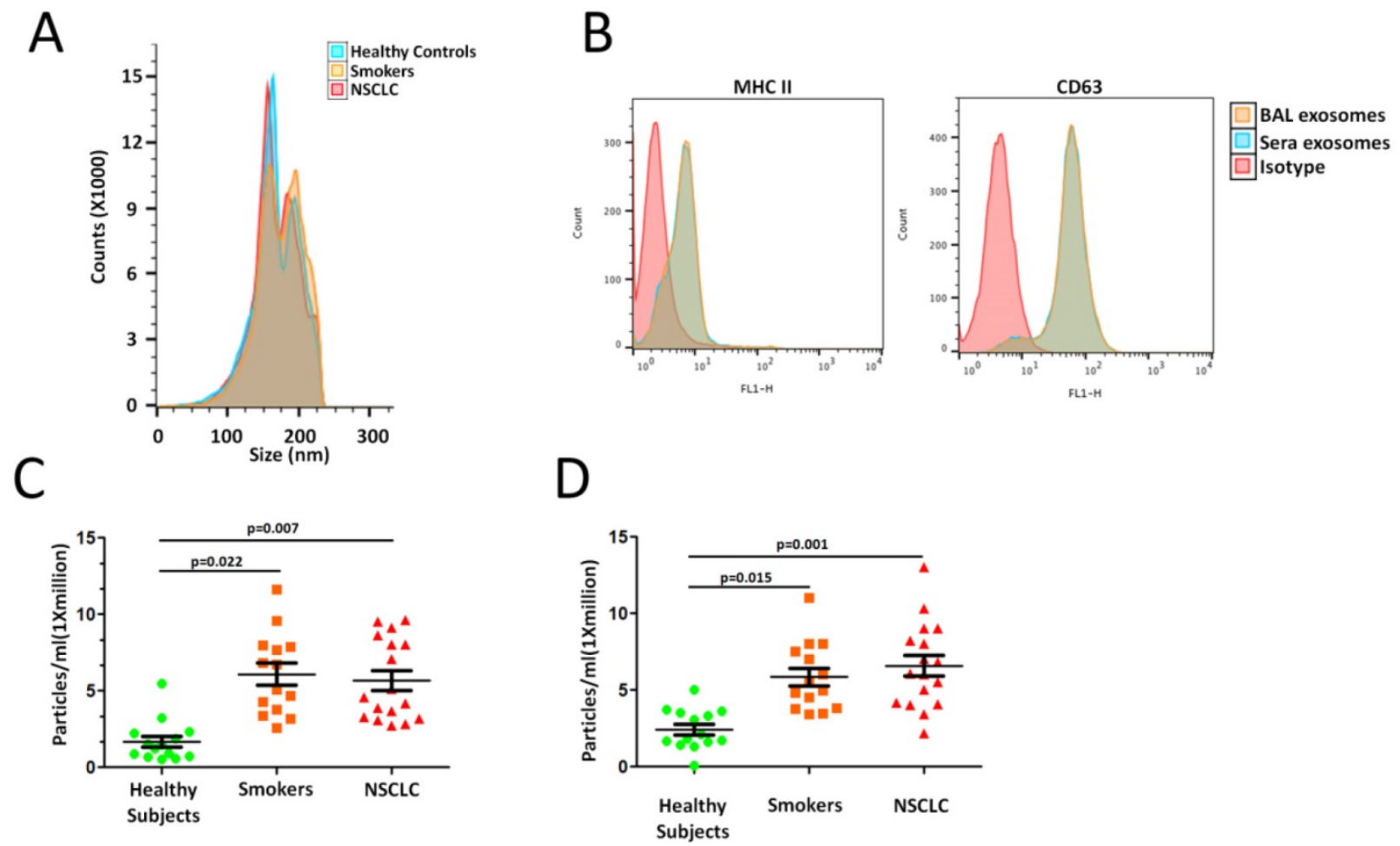

Figure 1. Higher EV concentration with smoking. A. Exosomal phenotyped EVs were examined by flow cytometric analysis. B. Cross-sectional cohort analysis of EV concentration in 14 smokers and 17 NSCLC patients compared to 14 healthy subjects. C. EVs concentrations when isolated by differential ultracentrifugation protocols. 
ways were significantly enriched $(p<0.05$, FDR corrected) among differentially expressed vesicle miRNAs. Among them, Proteoglycans in cancer $(p=7.197 \mathrm{E}-13)$, Fatty acid biosynthesis $(p=5.120 \mathrm{E}-10)$, ErbB ( $p=7.282 E-09)$, Hippo $(p=2.989 E-07)$, TGF-beta $(p=6.440 E-07)$, Pluripotency of stem cells $(p=9.569$ E-07), Pathways in cancer $(p=8.143 E-06)$, Wnt $(p=$ 2.151E-05), Renal cell carcinoma $(p=4.700 \mathrm{E}-05)$, Regulation of actin cytoskeleton ( $p=4.976 \mathrm{E}-05)$, Ubiquitin mediated proteolysis $(p=1.023 \mathrm{E}-04), \operatorname{Rap} 1 \quad(\mathrm{p}=1.353$ E-04), Glioma $(p=1.353 E-04)$ AMPK $(p=5.012 E-04)$, FoxO ( $p=5.472 \mathrm{E}-04)$, and Ras $(p=5.472 \mathrm{E}-04)$ were significantly enriched (Table 2), suggesting that smoking induced exosomal miRNAs may dysregulate specific biologic pathways, which predisposed to NSCLC.

Table 1. Exosomal microRNAs were differentially expressed in smokers and lung cancer patients.

\begin{tabular}{lllll}
\hline & $\begin{array}{l}\text { Smokers vs. } \\
\text { Controls }\end{array}$ & $\begin{array}{l}\text { Smokers vs. } \\
\text { Controls }\end{array}$ & $\begin{array}{l}\text { NSCLC vs. } \\
\text { Smokers }\end{array}$ & $\begin{array}{l}\text { NSCLC vs. } \\
\text { Smokers }\end{array}$ \\
\cline { 2 - 5 } & Fold change & $\begin{array}{l}\text { Adjusted } \\
\text { p-value }\end{array}$ & Fold change & $\begin{array}{l}\text { Adjusted } \\
\text { p-value }\end{array}$ \\
\hline miR-29a & 5.924 & 0.00117 & 1.954 & 0.00651 \\
miR-107 & 25.386 & 0.00753 & 2.502 & 0.00693 \\
miR-140-3p & 3.373 & 0.00776 & 4.415 & 0.00758 \\
\hline
\end{tabular}

\begin{tabular}{|c|c|c|c|c|}
\hline & $\begin{array}{l}\text { Smokers vs. } \\
\text { Controls }\end{array}$ & $\begin{array}{l}\text { Smokers vs. } \\
\text { Controls }\end{array}$ & $\begin{array}{l}\text { NSCLC vs. } \\
\text { Smokers }\end{array}$ & $\begin{array}{l}\text { NSCLC vs. } \\
\text { Smokers }\end{array}$ \\
\hline miR-Name & Fold change & $\begin{array}{l}\text { Adjusted } \\
\text { p-value }\end{array}$ & Fold change & $\begin{array}{l}\text { Adjusted } \\
\text { p-value }\end{array}$ \\
\hline miR-15a-5p & 3.117 & 0.00530 & 5.054 & 0.00832 \\
\hline miR-16-5p & 2.752 & 0.00498 & 1.584 & 0.00856 \\
\hline miR-20a-5p & 4.272 & 0.00984 & 2.971 & 0.00856 \\
\hline miR-19a-3p & 7.593 & 0.00283 & 1.555 & 0.00896 \\
\hline miR-126 & 19.105 & 0.00434 & 0.969 & 0.00901 \\
\hline miR-494 & 2.527 & 0.00183 & 0.550 & 0.00920 \\
\hline miR-572 & 3.013 & 0.00279 & 3.165 & 0.00977 \\
\hline miR-485-3p & 4.099 & 0.00068 & 3.484 & 0.01044 \\
\hline miR-124-3p & 6.447 & 0.04052 & 2.709 & 0.01051 \\
\hline miR-652-5p & 8.507 & 0.00990 & 1.005 & 0.01184 \\
\hline miR-934 & 5.409 & 0.00632 & 1.482 & 0.01249 \\
\hline miR-936 & 2.534 & 0.00737 & 1.621 & 0.01374 \\
\hline miR-939-5p & 3.331 & 0.00361 & 2.423 & 0.01441 \\
\hline miR-141-3p & 2.967 & 0.00034 & 7.238 & 0.01452 \\
\hline miR-17-5p & 3.319 & 0.00367 & 1.797 & 0.01453 \\
\hline miR-200a-3p & 8.561 & 0.00136 & 1.086 & 0.01798 \\
\hline $\operatorname{miR}-21-5 p$ & 4.142 & 0.00709 & 0.873 & 0.01933 \\
\hline miR-30b-5p & 5.924 & 0.00789 & 2.310 & 0.01988 \\
\hline miR-202 & 0.093 & 0.00201 & 0.149 & 0.02065 \\
\hline miR-548 & 0.25 & 0.00794 & 0.155 & 0.02155 \\
\hline miR-3713 & 0.066 & 0.00503 & 0.059 & 0.02222 \\
\hline $\operatorname{miR}-4768$ & 0.358 & 0.00065 & 0.492 & 0.02229 \\
\hline $\operatorname{miR-127}$ & 0.127 & 0.00442 & 0.168 & 0.02281 \\
\hline miR-146b & 0.254 & 0.00921 & 0.176 & 0.02477 \\
\hline miR-196a-5p & 0.039 & 0.00665 & 0.130 & 0.02683 \\
\hline miR-133 & 0.108 & 0.00482 & 0.149 & 0.03087 \\
\hline miR-590 & 0.284 & 0.00677 & 0.085 & 0.03176 \\
\hline miR-93-5p & 0.143 & 0.01742 & 0.163 & 0.00065 \\
\hline
\end{tabular}

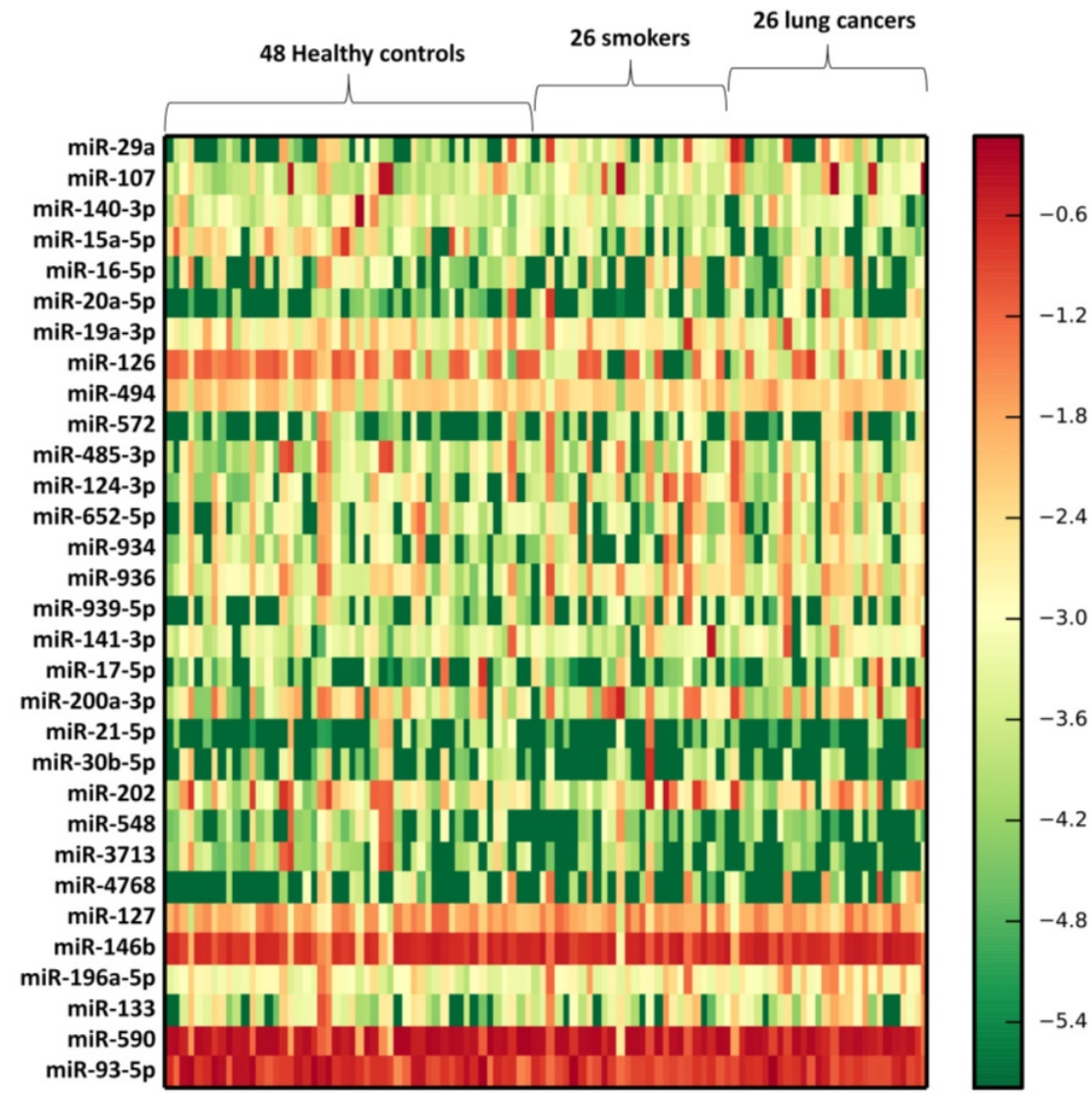

Figure 2. Heatmap of BAL exosomal differential miRNA profiles in smokers and NSCLC patients. Heatmap representation of the mean fold change in smokers and NSCLC patients related differential miRNA signature. Two-dimensional grid matrix displaying 31 exosomal miRNAs in BAL was obtained by the functional heat-map in R. Columns refer to time course comparison: 48 healthy controls, 26 smokers and 26 NSCLC patients. Rows stand for the 31 differential miRNAs. Each entry of the grid refers to relative fold ( $\log 2)$ between the expression level of a given exosomal miRNA. The color of each entry is determined by the value of that fold difference, ranging from green (negative values) to red (positive values). 
Table 2. Biologic pathways enriched by differentially expressed smoking specific miRNAs.

\begin{tabular}{|c|c|c|c|}
\hline KEGG pathway & p-value & \#genes & \#miRNAs \\
\hline Proteoglycans in cancer (hsa05205) & 7.197E-13 & 110 & 21 \\
\hline Fatty acid biosynthesis (hsa00061) & $5.120 \mathrm{E}-10$ & 5 & 5 \\
\hline ErbB signaling pathway (hsa04012) & 7.282E-09 & 56 & 21 \\
\hline Hippo signaling pathway (hsa04390) & $2.989 \mathrm{E}-07$ & 76 & 21 \\
\hline TGF-beta signaling pathway (hsa04350) & $6.440 \mathrm{E}-07$ & 47 & 19 \\
\hline $\begin{array}{l}\text { Signaling pathways regulating pluripotency of } \\
\text { stem cells (hsa04550) }\end{array}$ & $9.569 \mathrm{E}-07$ & 74 & 21 \\
\hline Pathways in cancer (hsa05200) & 8.143E-06 & 182 & 21 \\
\hline Wnt signaling pathway (hsa04310) & 2.151E-05 & 74 & 21 \\
\hline Renal cell carcinoma (hsa05211) & $4.700 \mathrm{E}-05$ & 41 & 20 \\
\hline Regulation of actin cytoskeleton (hsa04810) & 4.976E-05 & 106 & 21 \\
\hline Ubiquitin mediated proteolysis (hsa04120) & $1.023 \mathrm{E}-04$ & 71 & 21 \\
\hline Rap1 signaling pathway (hsa04015) & 1.353E-04 & 103 & 21 \\
\hline Glioma (hsa05214) & 1.353E-04 & 35 & 21 \\
\hline AMPK signaling pathway (hsa04152) & 5.012E-04 & 64 & 20 \\
\hline FoxO signaling pathway (hsa04068) & 5.472E-04 & 69 & 20 \\
\hline Ras signaling pathway (hsa04014) & 5.472E-04 & 105 & 21 \\
\hline Focal adhesion (hsa04510) & $6.650 \mathrm{E}-04$ & 100 & 21 \\
\hline mTOR signaling pathway (hsa04150) & 7.077E-04 & 37 & 18 \\
\hline Gap junction (hsa04540) & $7.258 \mathrm{E}-04$ & 45 & 19 \\
\hline Thyroid hormone signaling pathway (hsa04919) & $8.249 \mathrm{E}-04$ & 56 & 21 \\
\hline Neurotrophin signaling pathway (hsa04722) & 8.352E-04 & 65 & 20 \\
\hline Prolactin signaling pathway (hsa04917) & $9.840 \mathrm{E}-04$ & 37 & 18 \\
\hline Estrogen signaling pathway (hsa04915) & $9.963 \mathrm{E}-04$ & 45 & 20 \\
\hline Endocytosis (hsa04144) & $1.485 \mathrm{E}-03$ & 96 & 22 \\
\hline Circadian rhythm (hsa04710) & $1.690 \mathrm{E}-03$ & 22 & 15 \\
\hline Biotin metabolism (hsa00780) & $1.750 \mathrm{E}-03$ & 2 & 3 \\
\hline cGMP-PKG signaling pathway (hsa04022) & $1.750 \mathrm{E}-03$ & 79 & 20 \\
\hline Prostate cancer (hsa05215) & $1.829 \mathrm{E}-03$ & 46 & 19 \\
\hline Hedgehog signaling pathway (hsa04340) & 2.952E-03 & 30 & 15 \\
\hline MAPK signaling pathway (hsa04010) & 3.214E-03 & 114 & 20 \\
\hline $\begin{array}{l}\text { Adrenergic signaling in } \\
\text { cardiomyocytes (hsa04261) }\end{array}$ & 3.596E-03 & 65 & 20 \\
\hline Sphingolipid signaling pathway (hsa04071) & $3.858 \mathrm{E}-03$ & 57 & 19 \\
\hline Non-small cell lung cancer (hsa05223) & 4.639E-03 & 30 & 20 \\
\hline PI3K-Akt signaling pathway (hsa04151) & $4.970 \mathrm{E}-03$ & 146 & 21 \\
\hline Viral carcinogenesis (hsa05203) & 7.453E-03 & 81 & 20 \\
\hline Oxytocin signaling pathway (hsa04921) & 7.453E-03 & 76 & 21 \\
\hline Insulin signaling pathway (hsa04910) & $8.038 \mathrm{E}-03$ & 67 & 20 \\
\hline $\begin{array}{l}\text { Glycosaminoglycan biosynthesis - heparan } \\
\text { sulfate / heparin (hsa00534) }\end{array}$ & $8.620 \mathrm{E}-03$ & 12 & 15 \\
\hline Cell cycle (hsa04110) & $8.620 \mathrm{E}-03$ & 57 & 20 \\
\hline cAMP signaling pathway (hsa04024) & $8.620 \mathrm{E}-03$ & 90 & 21 \\
\hline Chronic myeloid leukemia (hsa05220) & $1.432 \mathrm{E}-02$ & 38 & 20 \\
\hline Adherens junction (hsa04520) & 1.547E-02 & 37 & 21 \\
\hline Melanoma (hsa05218) & $1.685 \mathrm{E}-02$ & 36 & 19 \\
\hline Amphetamine addiction (hsa05031) & $1.685 \mathrm{E}-02$ & 31 & 21 \\
\hline Melanogenesis (hsa04916) & 1.794E-02 & 47 & 18 \\
\hline Choline metabolism in cancer (hsa05231) & $1.953 \mathrm{E}-02$ & 50 & 20 \\
\hline T cell receptor signaling pathway (hsa04660) & $2.128 \mathrm{E}-02$ & 50 & 20 \\
\hline HIF-1 signaling pathway (hsa04066) & 2.981E-02 & 49 & 20 \\
\hline
\end{tabular}

Messenger RNA transcripts were differentially expressed in lung exosomes in smokers and NSCLC

We then employed quantitative RT-PCR to detect the expression of mRNA transcripts isolated from exosomes. Nine potential BAL biomarkers in lung cancers, including epidermal growth factor receptor (EGFR), kirsten ras (KRAS), anaplastic lymphoma kinase (ALK), MET, liver kinase B1 (LKB1), B-type raf kinase (BRAF), catalytic subunit of phosphoinositide 3-kinase (PIK3CA), RET, and c-ros oncogene 1 (ROS1), were selected for quantitative RT-PCR experiments using BAL exosomal RNA from
26 smokers, 26 NSCLC patients, and 48 healthy controls. EGFR, KRAS, ALK, MET, LKB1, PIK3CA, and ROS1 were found to be present and significantly over expressed in smokers and NSCLC lung exosome (Figure 3).

\section{Long non-coding RNAs were differentially expressed in lung exosomes isolated from smokers and NSCLC}

Further, we attempted to examine the presence of long non-coding RNAs (lncRNAs) in exosome and elucidate their roles in smokers and NSCLC patients. We then employed quantitative RT-PCR to detect the expression of lncRNAs isolated from lung exosomes. Nine potential lung cancers associated lncRNAs, including MALAT1, HOTAIR, HOTTIP, AGAP2-AS1, ATB, TCF7, FOXD2-AS1, HOXA11-AS, PCAF1, and BCAR4 were selected for quantitative RT-PCR experiments in 26 smokers, 26 NSCLC patients, and 48 healthy subjects. MALAT1, AGAP2-AS1, ATB, TCF7, FOXD2-AS1, HOXA11-AS, PCAF1, and BVAR4 were found to be present on the exosomes and significantly over expressed in smokers and NSCLC patients (Figure 4).

\section{Smoking alters tumor associated antigens in EVs}

Tumor associated antigens are associated with NSCLC [16]. Therefore, we analyzed for dysregulation of HLA-class I, AKAP4, BAGE, PD-L1, MAGE-3, and Annexin-A2 in lung exosomes of 5 healthy controls, 5 smokers, and 5 NSCLC patients. Clinicopathologic characteristics of smokers and NSCLC patients are summarized in Supplementary Table 3. We used western-blot to test proteins from lysed EVs. Significant elevation of HLA-class I, BAGE, PD-L1, and Annexin-A2 protein level in smokers and NSCLC patients compared to those from healthy subjects (Figure 5). However, no difference was observed in expression of AKAP4 and MAGE-3 protein levels among the three groups (Figure 5). These data indicated that EV cargo is affected by smoking and may reflect the tumor associated antigens presentation in lung exosomes from smokers and NSCLC.

\section{Discussion}

In this study, for the first time, we have verified the presence of EVs isolated from lung fluid from smokers and NSCLC patients. Smoking is associated with higher levels of local site EVs in the lung. Further, we revealed alterations in the vesicle miRNAs, mRNAs, lncRNAs profiles from smokers and NSCLC, suggesting the importance of EV contents in NSCLC development. 
Somatic mutations in EGFR, KRAS, ALK, MET, LKB1, BRAF, PIK3CA, RET, and ROS1 genes are frequently detected in lung cancers. EGFR is known to be a good tumor target in lung cancers. KRAS plays the critical role in growth factor receptor signaling and KRAS gene mutations are usually detected in lung adenocarcinomas [17]. Both EGFR and KRAS genes are responsible for encoding proteins that are embedded in cellular membranes. EGFR or KRAS mutations caused the protein production that is constantly turned on. If these mutations occur in lungs, lung cancer would develop. Rikova et al., [18] reported the MET receptor as the most important members of tyrosinated phosphopeptides in NSCLC tissue samples. V600E (50\%), G469A (39\%), and D594G (11\%) on the BRAF kinase were identified as mutations in lung adenocarcinomas [19]. EML4-ALK fusion was found in NSCLC by Soda et al.[20] in 2007 as a novel potential oncogenic kinase. In our current study, we found that EGFR, KRAS, ALK, MET, LKB1, PIK3CA, and ROS1 were present on the exosome surface and further over expressed in lung exosome from smokers and NSCLC patients (Figure 2). Therefore, smoking induced exosomal mRNA molecules may play an important role in NSCLC.
LncRNA MALAT1 promoted brain metastasis by inducing EMT in lung cancer [21, 22], which indicated a poor prognosis in NSCLC. Up regulation of lncRNA HOTAIR was reported to enhance aggressive tumor phenotypes in human NSCLC [23]. AGAP2-AS1 negatively controled lung cancer development by inhibiting LATS2 and KLF2 expression [24]. IncRNA ATB over expression demonstrated a poor prognosis in NSCLC [25]. TCF7 enhanced invasion by increasing EpCAM expression [26]. FOXD2-AS1 promoted NSCLC cell growth by Wnt/ $\beta$-catenin pathway [27]. HOXA11-AS regulated DOCK8 and TGF-beta pathway, further promoted NSCLC development [28]. PCAT-1 played as an oncogenic molecule in NSCLC progression [29]. Increased expression of long non-coding RNA BCAR4 is predictive of poor prognosis in NSCLC patients [30]. In this study, we, for the first time, found that MALAT1, AGAP2-AS1, ATB, TCF7, FOXD2-AS1, HOXA11-AS, PCAF1, and BVAR4 were present on the exosomes and significantly higher expressed in smokers and NSCLC patients (Figure 4). These results demonstrated that smoking dysregulated exosomal lncRNA molecules, which may predispose to NSCLC.


Figure 3. Quantitative RT-PCR experiments were performed on EGFR, KRAS, ALK, MET, LKB1, BRAF, PIK3CA, RET, and ROS1 mRNAs by using BAL exosomal RNA isolated from 26 smokers, 26 NSCLC patients, and 48 healthy controls. 



Figure 4. Quantitative RT-PCR experiments were performed on nine IncRNAs including MALAT1, HOTAIR, HOTTIP, AGAP2-AS1, ATB, TCF7, FOXD2-AS1, HOXA11-AS, PCAFI, and BCAR4 by using BAL exosomal RNA isolated from 26 smokers, 26 NSCLC patients, and 48 healthy controls.

$\begin{array}{llllllllllllllll}C 1 & C 2 & \text { C3 } & \text { C4 } & \text { C5 } & \text { S1 } & \text { S2 } & \text { S3 } & \text { S4 } & \text { S5 } & \text { T1 } & \text { T2 } & \text { T3 } & \text { T4 } & \text { T5 }\end{array}$

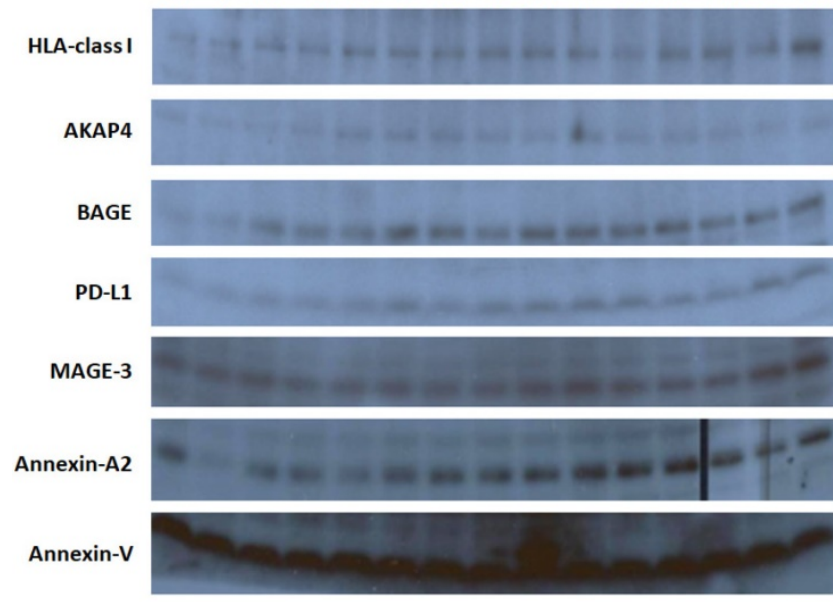

Figure 5. Western blot to test HLA-class I, AKAP4, BAGE, PD-L1, MAGE-3, and Annexin-A2 protein levels in lung exosomes isolated from 5 healthy controls, 5 smokers, and 5 NSCLC patients. Annexin- $V$ was loaded as a positive control.

Tumor associated antigens (TAAs) are thought to be an ideal target for cancer immunotherapy [31, 32]. Yasuda et al., identified a tumour associated antigen, annexin A2, in lung cancer patients with asbestos exposure [33]. They further found that the immune response in lung cancer was modulated by annexin A2 and it was also associated with high circulating levels of an inflammatory cytokine IL-6.
Mirandola et al [34] discovered that Sperm protein 17, AKAP4 and Pituitary tumor transforming gene 1 were potential immunotherapeutic targets in lung cancer patients. BAGE and MAGE-3 are expressed in lung cancer tissues [35]. MAGE-3 is aberrantly expressed in a wide variety of tumors, including NSCLC. Several CD8 ${ }^{+} \mathrm{T}$-cell epitopes of MAGE-3 have been identified in vitro [35]. Cancer cells can up regulate the surface expression of programmed death-ligand 1 (PD-L1), further interacts with programmed death-1 (PD-1) receptor on $\mathrm{T}$ cells to elicit the immune checkpoint response [36]. Exosomal PD-L1 carries active defense function to suppress $\mathrm{T}$ cell killing of breast cancer cells [37]. In this study, we found that HLA-class I, BAGE, PD-L1, and Annexin-A2 protein levels are over expressed in smokers and NSCLC patients (Figure 5). These data indicated that EV harbors tumor associated antigens presentation in smokers and NSCLC.

There are still some limitations. Sample size is small because obtaining more blood samples with inclusion criteria in this study is a challenge in our center. Results have to be validated in a larger sample-size cohort including potential confounding clinical variables. Furthermore, there was no functional investigation of the identified miRNAs. 
In conclusion, this pilot study demonstrated that smoking induced lung EVs in smokers and NSCLC patients. Furthermore, vesicle miRNAs, mRNAs, lncRNAs and protein presentations were dysregulated in smoking and NSCLCs. We propose that smoking induced exosomes carried tumor associated miRNAs, mRNAs, lncRNAs and tumor associated antigens, may participate in lung cancer development.

\section{Abbreviations}

EVs: Extracellular vesicles; NSCLC: non-small cell lung cancer; miRNAs: microRNAs; lncRNAs: long non-coding RNAs; BAL: bronchoalveolar lavage; NTA: nanoparticle tracking analysis; KEGG: Kyoto Encyclopedia of Genes and Genomes; EGFR: epidermal growth factor receptor; KRAS: kirsten ras; ALK: anaplastic lymphoma kinase; LKB1: liver kinase B1; BRAF: B-type raf kinase; PIK3CA: catalytic subunit of phosphoinositide 3-kinase; ROS1: c-ros oncogene 1.

\section{Supplementary Material}

Supplementary tables.

http://www.jcancer.org/v10p3435s1.pdf

\section{Acknowledgements}

This study is supported by the Natural Science Foundation of Hubei Province, China (Grant No. 2018 CFB590 to G.Y.) and the Natural Science Foundation of China (NSFC grant 81700091 to F.W.).

\section{Authors' contributions}

FW, ZYY, LY, and JSF performed the experiments and data analysis. JJX, YJ, JZY, and DZ collected the subject's samples and clinical data. GHY conceived the study and supervised this study. All the authors contributed to the manuscript writing and revision.

\section{Competing Interests}

The authors have declared that no competing interest exists.

\section{References}

1. Morabia A, Wynder EL. Cigarette smoking and lung cancer cell types. Cancer. 1991; 68: 2074-8.

2. Matsuura K, Kudoh S, Yoshimura N, Kimura T, Mitsuoka S, Hirata K, et al. Smoking history and prior surgical resection predict sensitivity to gefitinib in advanced non-small-cell lung cancer. Osaka city medical journal. 2006; 52: 1-8.

3. Igawa S, Sasaki J, Otani S, Shirasawa M, Niwa H, Kusuhara S, et al. Smoking History as a Predictor of Pemetrexed Monotherapy in Patients with Non-Squamous Non-Small Cell Lung Cancer. Oncology. 2016; 91: 41-7.

4. Gadgeel SM. Smoking history: tumor biology and clinical pharmacokinetic implications in patients with lung cancer. Clinical lung cancer. 2006; 7: 369-70.

5. Hori M, Tanaka H, Wakai K, Sasazuki S, Katanoda K. Secondhand smoke exposure and risk of lung cancer in Japan: a systematic review and meta-analysis of epidemiologic studies. Japanese journal of clinical oncology. 2016; 46: 942-51.

6. Brennan P, Buffler PA, Reynolds P, Wu AH, Wichmann HE, Agudo A, et al. Secondhand smoke exposure in adulthood and risk of lung cancer among never smokers: a pooled analysis of two large studies. International journal of cancer. 2004; 109: 125-31.
7. Yoon YJ, Kim OY, Gho YS. Extracellular vesicles as emerging intercellular communicasomes. BMB reports. 2014; 47: 531-9.

8. Willms E, Johansson HJ, Mager I, Lee Y, Blomberg KE, Sadik M, et al. Cells release subpopulations of exosomes with distinct molecular and biological properties. Scientific reports. 2016; 6: 22519.

9. Li M, Yu D, Williams KJ, Liu ML. Tobacco smoke induces the generation of procoagulant microvesicles from human monocytes/macrophages. Arteriosclerosis, thrombosis, and vascular biology. 2010; 30: 1818-24

10. Grant R, Ansa-Addo E, Stratton D, Antwi-Baffour S, Jorfi S, Kholia S, et al. A filtration-based protocol to isolate human plasma membrane-derived vesicles and exosomes from blood plasma. Journal of immunological methods. 2011; 371: 143-51.

11. Casey RG, Joyce M, Roche-Nagle G, Cox D, Bouchier-Hayes DJ. Young male smokers have altered platelets and endothelium that precedes atherosclerosis. The Journal of surgical research. 2004; 116: 227-33.

12. Freeman DW, Noren Hooten N, Eitan E, Green J, Mode NA, Bodogai M, et al. Altered Extracellular Vesicle Concentration, Cargo and Function in Diabetes Mellitus. Diabetes. 2018.

13. Vlachos IS, Zagganas K, Paraskevopoulou MD, Georgakilas G, Karagkouni D, Vergoulis T, et al. DIANA-miRPath v3.0: deciphering microRNA function with experimental support. Nucleic acids research. 2015; 43: W460-6.

14. Lotvall J, Hill AF, Hochberg F, Buzas EI, Di Vizio D, Gardiner C, et al. Minimal experimental requirements for definition of extracellular vesicles and their functions: a position statement from the International Society for Extracellular Vesicles. Journal of extracellular vesicles. 2014; 3: 26913.

15. Kowal J, Arras G, Colombo M, Jouve M, Morath JP, Primdal-Bengtson B, et al. Proteomic comparison defines novel markers to characterize heterogeneous populations of extracellular vesicle subtypes. Proceedings of the National Academy of Sciences of the United States of America. 2016; 113: E968-77.

16. Yasumoto K, Hanagiri T, Takenoyama M. Lung cancer-associated tumor antigens and the present status of immunotherapy against non-small-cell lung cancer. General thoracic and cardiovascular surgery. 2009; 57: 449-57.

17. Aviel-Ronen S, Blackhall FH, Shepherd FA, Tsao MS. K-ras mutations in non-small-cell lung carcinoma: a review. Clinical lung cancer. 2006; 8: 30-8

18. Rikova K, Guo A, Zeng Q, Possemato A, Yu J, Haack H, et al. Global survey of phosphotyrosine signaling identifies oncogenic kinases in lung cancer. Cell. 2007; 131: 1190-203.

19. Paik PK, Arcila ME, Fara M, Sima CS, Miller VA, Kris MG, et al. Clinical characteristics of patients with lung adenocarcinomas harboring BRAF mutations. Journal of clinical oncology : official journal of the American Society of Clinical Oncology. 2011; 29: 2046-51.

20. Soda M, Choi YL, Enomoto M, Takada S, Yamashita Y, Ishikawa S, et al. Identification of the transforming EML4-ALK fusion gene in non-small-cell lung cancer. Nature. 2007; 448: 561-6.

21. Schmidt LH, Spieker T, Koschmieder S, Schaffers S, Humberg J, Jungen D, et al. The long noncoding MALAT-1 RNA indicates a poor prognosis in non-small cell lung cancer and induces migration and tumor growth. Journal of thoracic oncology : official publication of the International Association for the Study of Lung Cancer. 2011; 6: 1984-92.

22. Shen L, Chen L, Wang Y, Jiang X, Xia H, Zhuang Z. Long noncoding RNA MALAT1 promotes brain metastasis by inducing epithelial-mesenchymal transition in lung cancer. Journal of neuro-oncology. 2015; 121: 101-8.

23. Nakagawa T, Endo H, Yokoyama M, Abe J, Tamai K, Tanaka N, et al. Large noncoding RNA HOTAIR enhances aggressive biological behavior and is associated with short disease-free survival in human non-small cell lung cancer. Biochemical and biophysical research communications. 2013; 436: 319-24.

24. Li W, Sun M, Zang C, Ma P, He J, Zhang M, et al. Upregulated long non-coding RNA AGAP2-AS1 represses LATS2 and KLF2 expression through interacting with EZH2 and LSD1 in non-small-cell lung cancer cells. Cell death \& disease. 2016; 7: e2225.

25. Ke L, Xu SB, Wang J, Jiang XL, Xu MQ. High expression of long non-coding RNA ATB indicates a poor prognosis and regulates cell proliferation and metastasis in non-small cell lung cancer. Clinical \& translational oncology : official publication of the Federation of Spanish Oncology Societies and of the National Cancer Institute of Mexico. 2017; 19: 599-605.

26. Wu J, Wang D. Long noncoding RNA TCF7 promotes invasiveness and self-renewal of human non-small cell lung cancer cells. Human cell. 2017; 30: 23-9.

27. Rong L, Zhao R, Lu J. Highly expressed long non-coding RNA FOXD2-AS1 promotes non-small cell lung cancer progression via Wnt/beta-catenin signaling. Biochemical and biophysical research communications. 2017; 484: 586-91.

28. Zhang $\mathrm{Y}, \mathrm{He} \mathrm{RQ}$, Dang $\mathrm{YW}$, Zhang $\mathrm{XL}$, Wang $\mathrm{X}$, Huang $\mathrm{SN}$, et al. Comprehensive analysis of the long noncoding RNA HOXA11-AS gene interaction regulatory network in NSCLC cells. Cancer cell international. 2016; 16: 89 .

29. Zhao B, Hou X, Zhan H. Long non-coding RNA PCAT-1 over-expression promotes proliferation and metastasis in non-small cell lung cancer cells. International journal of clinical and experimental medicine. 2015; 8: 18482-7.

30. Gong J, Zhang H, He L, Wang L, Wang J. Increased Expression of Long Non-Coding RNA BCAR4 Is Predictive of Poor Prognosis in Patients with Non-Small Cell Lung Cancer. The Tohoku journal of experimental medicine. 2017; 241: 29-34 
31. Simpson AJ, Caballero OL, Jungbluth A, Chen YT, Old LJ. Cancer/testis antigens, gametogenesis and cancer. Nature reviews Cancer. 2005; 5: 615-25.

32. Old LJ. Cancer/testis (CT) antigens - a new link between gametogenesis and cancer. Cancer immunity. 2001; 1: 1.

33. Yasuda M, Hanagiri T, Shigematsu Y, Onitsuka T, Kuroda K, Baba T, et al. Identification of a tumour associated antigen in lung cancer patients with asbestos exposure. Anticancer research. 2010; 30: 2631-9.

34. Mirandola L, Figueroa JA, Phan TT, Grizzi F, Kim M, Rahman RL, et al. Novel antigens in non-small cell lung cancer: SP17, AKAP4, and PTTG1 are potential immunotherapeutic targets. Oncotarget. 2015; 6: 2812-26.

35. Ho MY, Tang SJ, Sun KH, Yang W. Immunotherapy for lung cancers. Journal of biomedicine \& biotechnology. 2011; 2011: 250860.

36. Chen L, Han X. Anti-PD-1/PD-L1 therapy of human cancer: past, present, and future. The Journal of clinical investigation. 2015; 125: 3384-91.

37. Yang Y, Li CW, Chan LC, Wei Y, Hsu JM, Xia W, et al. Exosomal PD-L1 harbors active defense function to suppress $\mathrm{T}$ cell killing of breast cancer cells and promote tumor growth. Cell research. 2018. 